\title{
The State-of-the-Art of Collaborative Technologies for Initial Vocational Education: A Systematic Literature Review
}

\author{
Beat A. Schwendimann*1, Bram De Wever², Raija Hämäläinen ${ }^{3}$, \\ and Alberto A. P. Cattaneo ${ }^{4}$ \\ ${ }^{1}$ École Polytechnique Fédérale de Lausanne, 1015 Lausanne, Switzerland \\ ${ }^{2}$ Ghent University, Department of Educational Studies, B-9000 Gent, Belgium \\ ${ }^{3}$ Faculty of Education, FIN-40014, University of Jyväskylä, Finland \\ ${ }^{4}$ Swiss Federal Institute for Vocational Education and Training (SFIVET), Switzerland
}

Received: 19.12.2017; Accepted: 16.03.2018; Published: 26.04.2018

\begin{abstract}
Future workplaces require collaboration skills in which members of different work communities use technologies to solve complex problems. Vocational education and training (VET) programs need to meet the challenge to prepare students to be part of a competent workforce. Particularly initial vocational education is under pressure to develop learners collaboration skills and abilities. To date, however, no attempt has been made to perform a comprehensive review of the use of computer-supported collaborative learning (CSCL) technologies across different vocational education settings to account for contextual factors of VET.

In this systematic review, 26 published studies were analyzed with respect to their demographics, research methodology, use of technology, and measured outcomes.

This review illuminates that research on CSCL still leaves the vocational learning context as an under-represented field of study. At the same time, technologies offer a range of new types of learning possibilities for vocational education. As the direct result of that development, vocational education is increasingly taking place in new technology-enhanced learning settings.

Education can benefit from the opportunities of CSCL technologies, but on the other hand, such technologies create new challenges for facilitating vocational learning. There-
\end{abstract}

\footnotetext{
* Corresponding author: beat.schwendimann@gmail.com
}

ISSN: 2197-8646

http://www.ijrvet.net 
fore, this review also identifies three topic areas specific to vocational learning (collaborative writing-to-learn, simulations and game-like solutions, and tangible objects) and enumerates desirable lines for future research.

Keywords: VET, Vocational Education and Training, Initial Vocational Education and Training, Computer-Supported Collaborative Learning, CSCL, Review, TechnologyEnhanced Learning

\section{Introduction}

Collaboration has always been an important element of learning and working. However, what has changed throughout the past decade is the extent to which modern society and global working life require collaboration skills, particularly in technology-enhanced environments (Hämäläinen, Cincinnato, Malin, \& De Wever, 2014; Hämäläinen, De Wever, Malin, \& Cincinnato, 2015). Frameworks identifying the basic skills for 21st century learning emphasize the importance of collaboration for facing a constantly changing world (Partnership for 21st Century Skills, 2016; Trilling \& Fadel, 2009). Research on collaboration date back to earlier research on group-based learning, especially on cooperative learning (Qin, Johnson, \& Johnson, 1995). In cooperative learning a task is often divided into subtasks among learners and each of them is responsible for a share of the problem; in the end of problem-solving, these subtasks are combined into a joint output (Stahl, Koschmann, \& Suthers, 2006). Collaborative learning takes one step further by focusing on the potential that shared group processes have for learning by merging individual and social processes (Dillenbourg, 1999). Namely, according to Arvaja and colleagues (2007) collaboration refers to a shared knowledge construction in which it is not enough that participants cumulatively share knowledge together, but where the knowledge construction needs to be jointly put together based on others' ideas and thoughts. Current and future working life requires collaboration skills in which members of work communities work together, act effectively across different networks, and make decisions in teams (Lee, Huh, \& Reigeluth, 2015). Furthermore, collaborative learning is crucial when adapting and responding to new professional requirements of the radically changing workplace (Billett, 2014). In constantly transforming technology-intensive work environments, collaborative learning is also needed for developing working practices. As a direct result, advancing computer-supported collaborative learning (CSCL) (Cress, Stahl, Ludvigsen, \& Law, 2015) is of special importance to meet the requirements of the future working place.

Against this background, initial Vocational Education and Training (VET) is required not only to support the development of professional knowledge (i.e. specific contentknowledge on e.g. marketing, nursing, or electrical engineering) but also to prepare students for their future working lives (European Commission, 2013), allowing them to develop other kinds of skills. Initial VET (also called entry level training) can be defined as vocational education carried out in the initial education system, usually before entering working life (Maclean, \& Wilson, 2009). Stamm (2007) showed how vocational 
learners are qualified for professional life as they hold "practical intelligence", which includes both specialized knowledge in the professional domain and its application to practice. Practical intelligence articulates technical, specific, practical skills to "personal characteristics - such as reliability, willingness to take responsibility, social skills, ability to participate, teamwork/player, emotional intelligence, intuition" (Strahm, 2016, p. 43) and therefore constitutes a key factor to comply with the needed qualifications of the labor market. In this respect, VET is under pressure to enhance learners' collaboration skills.

Additionally, in view of preparing future workers for their jobs, using technologyenhanced learning can be an important driver ( Hämäläinen, Lanz, \& Koskinen, 2018). One the one hand, technology allows bringing more practice in the training of VET students. Especially in dual systems combining school-based and company-based tracks, technology can be exploited to reduce the gap that learners often perceive among learning locations (Taylor \& Freeman, 2011; Eteläpelto, 2008). On the other hand, technology can be used to support collaboration, together with the application and practice of the abovementioned practical intelligence skills and attitudes. Specific models to exploit both these affordances of technology in vocational education have been recently elaborated (Hämäläinen \& Cattaneo, 2015).

Therefore, it seems that CSCL may hold great promises for VET. The question is how the CSCL society responds to the needs of VET by meeting the challenges of developing and improving a broad range of collaboration skills needed in working life as well as using technology to cope with these challenges. So far, however, there is no comprehensive research on CSCL in VET. In 2012, the search term "vocational" in ijCSCL resulted in only three studies (Hämäläinen \& De Wever, 2013), which illustrates how critically underrepresented technology-supported vocational learning is. The interesting question for advancing VET involves what kind of new knowledge CSCL research produces on VET nowadays. To date, no attempt has been made to conduct a comprehensive review on CSCL across different vocational education settings. To contribute to this discussion, the aim of this paper is to provide a systematic review of studies that are focusing on initial VET within a CSCL context.

\section{Research Questions}

This paper offers a systematic literature review of the state of art and topic areas of research on computer-supported collaborative learning in the field of vocational education. The review focuses specifically on initial vocational education, rather than vocational or professional education in general. More specifically, the following research questions will be investigated:

1. What are the demographics of the selected studies on CSLC and initial VET (sample groups, countries, work domains)? 
2. What research methodologies were used in the selected studies on CSCL and initial VET (type of study, data sources, framework, actors and interactions, and technology design and usage)?

3. What are the measured outcomes on CSCL in initial VET (focus of analysis, forms of collaboration)?

4. What kinds of research topic areas can be identified applying collaborative technologies for initial vocational education?

\section{Methods}

First, to answer our quantitative research questions 1, 2, and 3, we have performed a systematic literature review following the guidelines proposed by Kitchenham and Charters (Kitchenham \& Charters, 2007). Second, to identify what kinds of topic areas emerge in research applying collaborative technologies for initial vocational education (research question 4), we conducted a thematic content analysis (Braun \& Clarke, 2006) of the resulting corpus of empirical articles, using a grounded theory methodology. The aim of the second phase was to identify emergent topic areas that represent findings shared across multiple studies.

\subsection{Paper Selection Processes}

This study selected relevant papers following a stepwise procedure consisting of nine steps. As the first step, we selected the databases in which to conduct or searches. We searched the following databases: Scopus ${ }^{1}$, ISI Web of knowledge ${ }^{2}$, LibHul ${ }^{3}$, ERIC 4 , Proquest Education Journal 5 , JSTOR 6 , Sciencedirect7 and Google Scholar ${ }^{8}$,

Second, we developed the search query. To perform the search, we broke the question down into the context (VET), the type of interaction (collaboration), and the technology. With regards to VET, the title, abstract, or keywords of the papers needed to include either "VET" (as an abbreviation) or "vocational" in combination with "education" or "training" (or both). In addition, with regards to collaborative learning, the term "collaborat*" was included. With regards to TEL, the following terms were included: "technolog*" or "online" or "web-based" or "computer*". Depending on the databases, search strings were slightly adapted. An example search string used for ISI web of knowledge is: " TS=(((VET OR Vocational) AND (education OR training) $)$ AND (collaborat* AND (computer* OR technolog*)))". For some databases, full-text papers

\footnotetext{
${ }^{1}$ Scopus: https://www.scopus.com/

${ }^{2}$ ISI Web of knowledge: http://apps.webofknowledge.com

${ }^{3}$ LibHub: http://www.libhub.org

${ }^{4}$ ERIC: https://eric.ed.gov/

${ }^{5}$ Proquest: http://www.proquest.com/products-services/pq_ed_journals.html

${ }^{6}$ JSTORhttp://www.jstor.org/

${ }^{7}$ Sciencedirect: http://www.sciencedirect.com

${ }^{8}$ Google Scholar: https://scholar.google.ch
} 
could be searched for these terms. Whenever possible (depending on the database), searches were restricted to peer-reviewed papers (e.g. ERIC and Proquest). Only papers written in English were included in the search. The searches were conducted during July and August, 2015. In the end, 823 items were selected (see Figure 1).

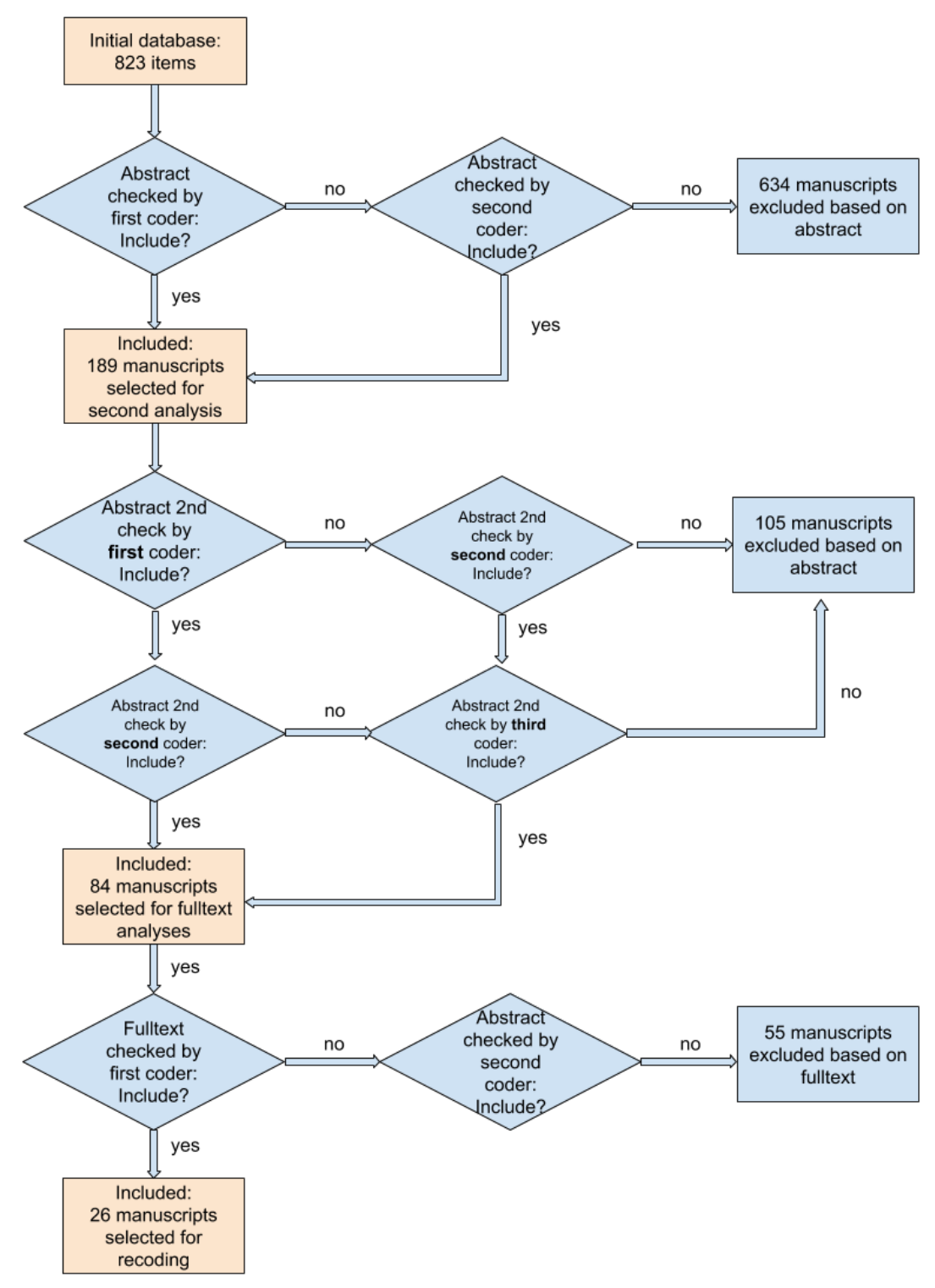

Figure 1: Paper selection process

In step three, we defined the exclusion criteria for manually screening the abstracts. These were discussed among the four authors, and when agreement was reached, they were operationalized in detail. Exclusion criteria were: after reading the abstract, there 
is no indication at all that the study is on (1) VET or (2) collaborative learning, or that the study has (3) empirical results and provided adequate descriptions of their methodology. This means that papers were excluded if they did not meet these three criteria.

In step four, all 823 abstracts were screened for inclusion or exclusion. This was done in two phases. First, a first coder (one of the authors of this manuscript) marked all abstracts for exclusion. Second, all abstracts marked for exclusion by the first coder, were randomly assigned to one of the three other authors, who served as a second coder. These were double-checked. If the second coder agreed on the exclusion, the item was excluded. If the second coder disagreed or doubted, the item remained in the database. Duplicate papers (same paper indexed in different databases) were merged. After this third step, 634 items of the 823 were excluded.

Fifth, after a first round of evaluating abstracts in step four, the exclusion criteria were further refined. We decided to keep the aforementioned exclusion criteria, but added that the abstract had to make clear that the study was focusing on initial VET, meaning that studies focusing on professional development, including, amongst others, teacher training, or medical training, were excluded.

The remaining 189 abstracts were re-coded in step six while keeping the additional exclusion criteria. In this step, we first made sure that all abstracts were coded independently by two coders (two randomly selected authors). Disagreements between coders were resolved with the help of a third coder (one randomly selected author out of the two remaining ones). This resulted in 68 abstracts to be included (both coders agreed not to exclude), 54 abstracts to be excluded (both coders agreed to exclude), and 67 abstracts to be double-checked by a third coder, as one of the initial coders in step six would include the paper, and the other one would exclude it. The third coder first coded these abstracts independently, and after that, decided to include or exclude the paper (after discussion with the other coders). When in doubt, the paper was included. In total, 84 papers were selected for full-text analysis.

In step seven, all papers were randomly divided among the four authors for a first full-text screening. Some papers were not available from journal websites (journal subscriptions) or databases. Online search engines were used to track them down, in combination with searches on social academic websites, such as researchgate and academia.edu, personal websites of the authors and direct emails to the authors.

During step eight, 55 out of the 84 selected papers for full-text analysis were excluded after reading the full-text version, based on exactly the same criteria. All of these exclusions were double checked by another coder, which was randomly selected from the remaining three authors. In this way, we derived our final selection of 26 articles. The selection process is depicted in Figure 1.

Last, the final selection of papers was analyzed by all four authors according to the following scheme to answer the research questions (see table 1 and figure 2). Discrepancies in codes were discussed and resolved among the four coders. Overall, discrepancies in this final selection step were rare. The codes distinguish demographics (sample sizes, countries, work domains), research methods (data collection, analysis focus), technology aspects (type, novelty, access), and the measured outcomes of the CSCL. 
Table 1: Recoding schema indicating how each of the variables was coded.

\begin{tabular}{ll}
\hline Variable & Coding \\
\hline Participants & Country and sample size (if multiple studies presented \\
& in the same paper, the sample sizes represents the \\
& sum) \\
\hline Work domain & Using the 22 domains distinguished by Jungo and \\
& Zihlmann (Zihlmann \& Jungo, 2015): (1) Nature \\
& (agriculture, forestry, animals, environment); (2) Food \\
& (food technology), (3) Gastronomy (Restaurants and \\
& hotels); (4) Textile (clothing design and production); \\
& (5) Beauty and sport (hair, make-up, sport); (6) \\
& Design and art (artist, designer, visual communica- \\
& tion); (7) Print (print shops); (8) Construction (build- \\
& ings, bridges, tunnels, streets, railways); (9) Building \\
& technology (tinsmith, plumber, janitor); (10) Wood \\
& (carpenter, furniture builder); (11) Vehicles (car me- \\
& chanic); (12) Electrotechnic (electrician); (13) Metal \\
& and machines (machine construction, foundry, smith, \\
& watchmaker); (14) Chemistry and physics (laboratory, \\
& chemical industry); (15) Planning (technical drawer); \\
& (16) Business administration; (17) Administration \\
& (civil servants, insurances, banks, tourism); (18) Lo- \\
gistics and traffic (logistics, security, police, military, & truck driver); (19) Information technology (software \\
developer, computer repairs); (20) Culture (journal- & ist, actor, musician, culture management); (21) Health \\
(nurses, paramedic, medical doctor, dentist); (22) Ed- & ucation and social (teacher, social worker, minister); \\
(23) General studies; (99) Multiple
\end{tabular}




\begin{tabular}{|c|c|}
\hline Focus on actors & $\begin{array}{l}\text { None provided }(0) \text {; learners }(1) \text {; teachers }(2) \text {; supervi- } \\
\text { sors }(3) \text {; multiple }(4)\end{array}$ \\
\hline Framework & $\begin{array}{l}\text { Scripting/scaffolding/orchestration (1); problem- } \\
\text { based learning (2); boundary-crossing (3); gam- } \\
\text { fication/ game-based learning (4); community of } \\
\text { practice/ social presence (5); reflection (6); self- } \\
\text { regulation/autonomous learning/ self-efficacy (7); } \\
\text { activity theory (8); peer tutoring (9). [Multiple } \\
\text { answers possible] }\end{array}$ \\
\hline $\begin{array}{l}\text { Novelty of technol- } \\
\text { ogy }\end{array}$ & $\begin{array}{l}\text { Existing technology (1); development and study of } \\
\text { new technology (2) }\end{array}$ \\
\hline Interaction & $\begin{array}{l}\text { Learner-learner (1); learner-teacher (2); learner- } \\
\text { supervisor }(3) \text {; teacher-teacher }(4) \text {; teacher-supervisor } \\
(5) \text {; supervisor-supervisor }(6) \text {. [Multiple answers pos- } \\
\text { sible]. }\end{array}$ \\
\hline Used technology & $\begin{array}{l}\text { Not specified (0); online platform (e.g. Moodle) (1); } \\
\text { serious game }(2) \text {; tangible ( } 3 \text {; ; augmented reality (4); } \\
\text { blog or wiki }(5) \text {. [Multiple answers possible]. }\end{array}$ \\
\hline Type of technology & $\begin{array}{l}\text { Not specified (0); mobile (1); desktop (2); web-based } \\
(3) \text {; hardware (e.g. Tinkerlamp) (4); multiple (5). } \\
\text { [Multiple answers possible]. }\end{array}$ \\
\hline
\end{tabular}

The quantitative analysis was followed by a qualitative thematic content analysis, applying a grounded theory methodology (RQ 4). In qualitative thematic analysis (Braun \& Clarke, 2006) there is particular concern with the reliability, firstly, in choosing the most relevant literature, and secondly in identifying topic areas reliably. To identify emerging topic areas across these studies, we used the grounded theory methodology. In analyzing the 26 research articles, we followed Aveyard's (2010) idea of explaining the differences and similarities in the different papers, rather than to simply summarizing them. The methodology allowed topic areas to emerge from the papers, rather than being predetermined through a theoretical framework or a hypothesis. Therefore, for each article, we developed a short summary, assigned keywords, and composed a short statement of the findings. Finally, we (four co-authors) engaged in a process of critical discussion of the emerging topic areas. 


\begin{tabular}{|c|c|c|c|c|c|c|c|c|c|c|c|c|}
\hline $\begin{array}{l}\frac{5}{0} \\
\stackrel{0}{+0} \\
\stackrel{5}{5}\end{array}$ & $\begin{array}{l}\vec{z} \\
\stackrel{2}{5} \\
0 \\
0\end{array}$ & $\begin{array}{l}\frac{5}{\pi} \\
\text { है } \\
\frac{0}{0} \\
\frac{1}{2} \\
\vdots \\
3\end{array}$ & $\begin{array}{l}\frac{N}{N} \\
\frac{\Delta}{0} \\
\frac{0}{E} \\
D \\
D\end{array}$ & 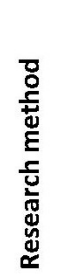 & 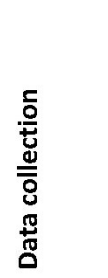 & $\begin{array}{l}\frac{n}{u} \\
\frac{0}{0} \\
\frac{n}{n} \\
\frac{n}{n} \\
\frac{c}{\alpha}\end{array}$ & 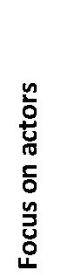 & 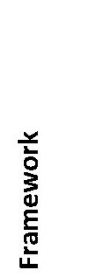 & 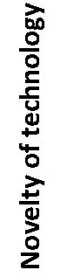 & 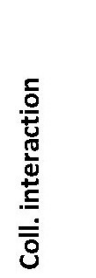 & 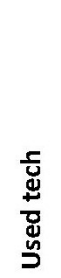 & 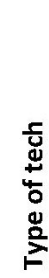 \\
\hline $\begin{array}{l}\text { (Minnaert, Boekaerts, de } \\
\text { Brabander, \& Opdenakker, 2011) }\end{array}$ & $\mathrm{NL}$ & 17 & 137 & 1 & $5 ; 6$ & 1 & 4 & 7 & 2 & 1 & 1 & 0 \\
\hline (Hämäläinen et al., 2008) & $\mathrm{FI}$ & 8 & 64 & 1 & $\begin{array}{l}1 ; 2 ; 4 ; \\
6\end{array}$ & 2 & 1 & $1 ; 4$ & 2 & 1 & 2 & 3 \\
\hline (Hämäläinen \& Oksanen, 2012b) & $\mathrm{FI}$ & 99 & 20 & 1 & $1 ; 4$ & 1 & 4 & $1 ; 4$ & 2 & $1 ; 2$ & 2 & 3 \\
\hline (Hämäläinen \& Oksanen, 2013) & $\mathrm{FI}$ & 23 & 30 & 1 & 1 & 1 & 4 & $1 ; 4$ & 2 & $1 ; 2$ & 2 & 3 \\
\hline (Motta et al., 2013) & $\mathrm{CH}$ & 16 & 186 & 2 & $6 ; 7$ & 2 & 1 & $\begin{array}{l}1 ; 7 ; 6 ; \\
9\end{array}$ & 1 & 1 & 5 & 3 \\
\hline $\begin{array}{l}\text { (Chanchalor \& Kammeungmai, } \\
\text { 2011) }\end{array}$ & $\mathrm{TH}$ & 12 & 92 & 2 & $\begin{array}{l}1 ; 2 ; 3 ; \\
6\end{array}$ & 3 & 1 & 0 & 1 & 1 & 1 & 0 \\
\hline (Hämäläinen, 2011) & $\mathrm{FI}$ & 8 & 48 & 1 & $\begin{array}{l}1 ; 2 ; 4 ; \\
6\end{array}$ & 3 & 1 & $1 ; 4$ & 2 & 1 & 2 & 3 \\
\hline (Schneider et al., 2011) & $\mathrm{CH}$ & 18 & 82 & 2 & $1 ; 3 ; 6$ & 3 & 1 & $\begin{array}{l}1 ; 4 ; 2 ; \\
3\end{array}$ & 2 & 1 & 3 & 4 \\
\hline (Valtonen et al., 2012) & $\mathrm{FI}$ & 99 & 33 & 1 & 7 & 1 & 1 & 6 & 1 & 1 & 1 & 0 \\
\hline (Jermann et al., 2009) & $\mathrm{CH}$ & 18 & 15 & 1 & 1 & 1 & 1 & $2 ; 3$ & 2 & 1 & 3 & 4 \\
\hline $\begin{array}{l}\text { (Beers, Kirschner, Boshuizen, \& } \\
\text { Gijselaers, 2007) }\end{array}$ & $\mathrm{NL}$ & 8 & 66 & 1 & $2 ; 6$ & 1 & 1 & $1 ; 2$ & 2 & 1 & 1 & 2 \\
\hline (Boldrini \& Cattaneo, 2014) & $\mathrm{CH}$ & 16 & 111 & 2 & $6 ; 3 ; 7$ & 3 & 1 & $1 ; 6$ & 1 & 1 & 1 & 2 \\
\hline (Inayat et al., 2013) & PK & 19 & 500 & 1 & $6 ; 7$ & 2 & 1 & 0 & 1 & 1 & 1 & 3 \\
\hline (Gavota et al., 2010) & $\mathrm{CH}$ & 99 & 51 & 2 & $6 ; 7$ & 2 & 1 & $1 ; 6 ; 9$ & 1 & $1 ; 2$ & 5 & 3 \\
\hline (Oksanen \& Hämäläinen, 2013a) & $\mathrm{FI}$ & 23 & 69 & 1 & 6 & 1 & 4 & $1 ; 4 ; 5$ & 2 & $1 ; 2$ & 2 & 3 \\
\hline (Oksanen \& Hämäläinen, 2012) & $\mathrm{FI}$ & 23 & 69 & 1 & 6 & 1 & 4 & $1 ; 4 ; 5$ & 2 & $1 ; 2$ & 2 & 3 \\
\hline (Oksanen \& Hämäläinen, 2013b) & $\mathrm{FI}$ & 23 & 86 & 1 & $1 ; 6$ & 1 & 1 & $1 ; 4 ; 5$ & 2 & 1 & 2 & 3 \\
\hline $\begin{array}{l}\text { (Cuendet, Dehler-Zufferey, } \\
\text { Ortoleva, \& Dillenbourg, 2015) }\end{array}$ & $\mathrm{CH}$ & 10 & 40 & 2 & $\begin{array}{l}1 ; 2 ; 3 ; \\
4 ; 5 ; 7\end{array}$ & 3 & 1 & $1 ; 3$ & 2 & $1 ; 2$ & 3 & 4 \\
\hline (Hämäläinen \& De Wever, 2013) & $\mathrm{FI}$ & 23 & 20 & 1 & 1 & 1 & 2 & $1 ; 4$ & 2 & 1 & 2 & 3 \\
\hline (Hämäläinen \& Cattaneo, 2015) & Mult & 99 & 115 & 1 & $1 ; 4$ & 1 & 4 & $1 ; 3$ & 2 & $1 ; 2 ; 3$ & 1 & 5 \\
\hline (Hämäläinen et al., 2008) & $\mathrm{FI}$ & 15 & 20 & 1 & $\begin{array}{l}1 ; 2 ; 5 ; \\
7\end{array}$ & 3 & 1 & $1 ; 4$ & 2 & 1 & 2 & 3 \\
\hline (Yang, 2015) & TW & 15 & 68 & 2 & 3 & 2 & 1 & $1 ; 4$ & 1 & 1 & 2 & 0 \\
\hline (Tielman et al., 2012) & $\mathrm{NL}$ & 16 & 29 & 1 & $4 ; 5$ & 1 & 2 & 0 & 1 & 0 & 0 & 0 \\
\hline (Ortoleva \& Bétrancourt, 2015) & $\mathrm{CH}$ & 21 & 42 & 2 & $3 ; 6 ; 7$ & 2 & 1 & $1 ; 7 ; 9$ & 1 & 1 & 5 & 3 \\
\hline (Sanz, 2010) & $\mathrm{CH}$ & 18 & 49 & 1 & $\begin{array}{l}1 ; 2 ; 3 \\
4 ; 5 ; 6\end{array}$ & 3 & 4 & $1 ; 3$ & 2 & $1 ; 2$ & 3 & 4 \\
\hline (Whicte, 2010) & $\mathrm{AU}$ & 23 & 20 & 1 & 5 & 1 & 4 & 0 & 1 & 1 & 1 & 5 \\
\hline
\end{tabular}

Figure 2: Overview of recoded papers 


\section{Results}

Quantitative results: inventory of article characteristics

What are the demographics of the selected studies on CSLC and initial VET (sample groups, countries, work domains)?

Sample sizes: Most studies are small scale (less than one hundred participants). Twelve studies have less than 50 participants, nine studies between 50 and 100, four between 100 and 200 and only one more than 200. This large study (Inayat, ul Amin, Inayat, \& Salim, 2013) included 500 participants. On average, studies included 79 participants (excluding the outlier 500-participants study, it goes down to 62); median 58 participants (excluding the outlier study, 51 participants). Overall, 2062 participants were included in the 26 papers.

Countries: Most papers have been produced in Switzerland (8 studies; 31\%; 605 participants total) and Finland (10 studies; 39\%; 459 participants total) (see Table 2). The contributions by Switzerland can be traced back to an extensive national research program supporting studies on CSCL in initial VET contexts. Most studies focus only on one particular country (25 papers), which take country-specificities into account. Only one paper includes multiple countries, but not as a comparison (Hämäläinen \& Cattaneo, 2015).

Work domain: In those papers focusing on only one profession, the work domain 'business administration' (4 studies; $15 \%$ ) has been most frequently studied, followed by logistics (3 studies; 12\%). All logistics and most of the business administration studies originated from Switzerland. Other professions include construction and planning (each 2 studies; $8 \%$ ). One study each referred to woodworkers, electronics, administration, and information technology. It is noteworthy that the majority of papers did not investigate a particular profession: Six studies (23\%) referred to 'general studies'. Additionally, $20 \%$ reported findings from multiple professions (see table 2). Two studies focused on health professions (one paper reporting on a single context and one on multiple professions). 
Table 2: Overview table (by country)

\begin{tabular}{|c|c|c|c|c|c|c|c|c|}
\hline & $\begin{array}{l}\text { The } \\
\text { Nether- } \\
\text { lands }\end{array}$ & $\begin{array}{l}\text { Finn- } \\
\text { land }\end{array}$ & $\begin{array}{l}\text { Switz- } \\
\text { erland }\end{array}$ & $\begin{array}{l}\text { Thai- } \\
\text { land }\end{array}$ & Taiwan & $\begin{array}{l}\text { Pakis- } \\
\text { tan }\end{array}$ & $\begin{array}{l}\text { Austra- } \\
\text { lia }\end{array}$ & $\begin{array}{l}\text { Multi- } \\
\text { ple }\end{array}$ \\
\hline Sample size & 232 & 459 & 576 & 92 & 68 & 500 & 20 & 115 \\
\hline $\begin{array}{l}\text { Existing } \\
\text { technology }\end{array}$ & $4 \%$ & $4 \%$ & $15 \%$ & $4 \%$ & $4 \%$ & $4 \%$ & $4 \%$ & \\
\hline $\begin{array}{l}\text { Novel tech- } \\
\text { nology }\end{array}$ & $8 \%$ & $35 \%$ & $15 \%$ & & & & & $4 \%$ \\
\hline Case study & $12 \%$ & $39 \%$ & $8 \%$ & & & $4 \%$ & $4 \%$ & $4 \%$ \\
\hline $\begin{array}{l}\text { Experimental } \\
\text { study }\end{array}$ & & & $23 \%$ & $4 \%$ & $4 \%$ & & & \\
\hline Construction & $4 \%$ & $4 \%$ & & & & & & \\
\hline Wood & & $4 \%$ & & & & & & \\
\hline Electrotechnic & & & & $4 \%$ & & & & \\
\hline Planning & & $4 \%$ & & & $4 \%$ & & & \\
\hline $\begin{array}{l}\text { Business ad- } \\
\text { ministration }\end{array}$ & $8 \%$ & & $8 \%$ & & & & & \\
\hline $\begin{array}{l}\text { Logistics and } \\
\text { traffic }\end{array}$ & & & $12 \%$ & & & & & \\
\hline $\begin{array}{l}\text { Information } \\
\text { technology }\end{array}$ & & & & & & $4 \%$ & & \\
\hline Health & & & $4 \%$ & & & & & \\
\hline $\begin{array}{l}\text { General } \\
\text { studies }\end{array}$ & & $19 \%$ & & & & & $4 \%$ & \\
\hline Multiple $(*)$ & & $12 \%$ & $4 \%$ & & & & & $4 \%$ \\
\hline
\end{tabular}

$\left({ }^{*}\right)$ : Four papers (Hämäläinen \& Oksanen, 2012; Valtonen et al., 2012; Gavota et al., 2010; Hämäläinen \& Cattaneo, 2015) reported on multiple professions. To avoid counting each profession separately, these papers are reported under 'Multiple'. 
What research methodologies were used in the selected studies on CSCL and initial VET (type of study, data collection, framework, actors and interactions, and technology design and usage)?

Type of study: About two thirds of the reviewed papers describes case studies (18 papers; $69 \%$ ); only about one third of the papers described experimental (or quasi-experimental studies (8 papers; 31\%). The countries with the most case studies are Finland (10 studies; $39 \%$ ) and the Netherlands (3 studies; $12 \%$ ); most experimental studies were conducted in Switzerland (6 studies; $23 \%$ ) (see Table 2).

Data collection: The three most popular methods for data collection were questionnaires (15 studies; $24 \%$ ), video (13 studies; $21 \%$ ), and artefacts (8 studies; $13 \%$ ). Other forms of data collection include fieldnotes and pre/posttests (each 7 studies; 11\%) as well as log data and interviews (each 6 studies; $10 \%)$. The majority of studies $(n=18)$ combined multiple forms of data collection.

Frameworks: We investigated if the reviewed studies used other common theoretical frameworks, beyond 'collaboration'. The most frequently used frameworks were 'scripting/ scaffolding/ orchestration' (20 studies; 39\%) and 'gamification/ game-based' (11 studies; 21\%). Other frameworks were 'boundary crossing' (5 studies; 10\%), 'reflection' (4 studies; $8 \%$ ), and 'peer tutoring', 'self-regulation', 'problem-based learning', and 'community of practice' (each 3 studies; $6 \%$ ). Two studies used only one framework; 13 studies used two frameworks, and 7 studies combined more than two frameworks. Four studies did not refer to a specific framework.

Actors and interactions: The majority of studies focused on students (16 studies; $62 \%$ ) and only two studies $(8 \%)$ on teachers. Eight $(31 \%)$ studies included both teachers and students. Similarly, most studies focused on (collaborative) student-student interactions (25 studies), eight studies on student-teacher interactions, and only one study included student-supervisor interactions (see Table 3).

Table 3: Focus of studies (by frequency and percent)

\begin{tabular}{lllll}
\hline & Case study & & $\begin{array}{l}\text { Experimental } \\
\text { study }\end{array}$ \\
\hline & Studentfocus & Teacherfocus & Both & Studentfocus \\
Process & $4(15 \%)$ & $2(8 \%)$ & $7(30 \%)$ & \\
Outcome & $2(8 \%)$ & & & $4(15 \%)$ \\
Both & $2(8 \%)$ & & $1(4 \%)$ & $4(15 \%)$ \\
\hline
\end{tabular}

Technology: When dealing with the application of vocational learning in CSCL, a distinction can be made between the design of new technologies to support learning and the use of existing technologies. Following this distinction, 10 studies explored how to make use of existing technologies (38\%). On the other hand, 16 studies presented novel tech- 
nologies $(62 \%)$ for enhancing learning in vocational contexts. The two most frequently used forms of collaborative technology are serious games (10 studies; 38\%) and online learning platforms (8 studies; 31\%). Serious games were typically based on authentic worklife situations and applied to learn holistic work processes, such as organizing an event (e.g. (Hämäläinen \& Oksanen, 2012a; Oksanen \& Hämäläinen, 2014), practicing electrical installation process of a house (Hämäläinen, 2011), solving tasks in the area of work safety (Hämäläinen, Oksanen, \& Häkkinen, 2008), or demonstrating the design process of surface treatment (Hämäläinen, 2008). Other technologies were tangibles (4 studies; $15 \%$ ) and blogs/wikis (3 studies; $12 \%$ ). The former were mainly used to support writing-to-learn collaborative activities to foster reflection on specific professional situations the participants personally lived at the workplace; this was applied to identify causes and possible solutions for faulty X-rays with dental assistants (Gavota, et al., 2010), to analyze critical incidents with social and health care assistant (Ortoleva \& Bétrancourt, 2015), and to gain deeper knowledge of the what and whys of the main steps of a procedure in the commerce field (Gavota et al., 2010; Motta et al, 2013). The latter were ad-hoc built solutions to address specific topics in the logisticians' (Schneider et al., 2011; Sanz, 2010; Jermann et al., 2009) and carpenters' (Cuendet et al., 2015) curricula. One study (Tielman, den Brok, Bolhuis, \& Vallejo, 2012) did not specify any particular technology as students accessed a range of different online sources. These observations align with the type of technology (see Table 4).

Thirteen studies described browser-based technologies (e.g. Moodle, Facebook, etc.) $(50 \%)$ and 2 studies (8\%) desktop software (see table 4). However, several studies did not specify how their technology was accessed (5 studies; 19\%) or mentioned multiple technologies without providing details $(2 ; 8 \%)$. Four studies used custom-built hardware (the previously mentioned studies on tangibles by the DUAL-T group) (15\%).

Novel technologies were mostly explored through case studies (14 studies; 54\%) (and 2 experimental studies, $8 \%$ ), while existing technologies were explored through experimental studies (6 studies; $23 \%$ ) and case studies (4 studies; 15\%).

Table 4: Technology access and type (by frequency and percent)

\begin{tabular}{llllll}
\hline & Not specified & Desktop & Webbased & Hardware & Multiple \\
\hline Online platform & $3(12 \%)$ & $2(8 \%)$ & $1(4 \%)$ & & $2(8 \%)$ \\
Serious game & $1(4 \%)$ & & $9(36 \%)$ & & \\
Tangibles & & & & $4(16 \%)$ & \\
Blogs/Wikis & & & $3(12 \%)$ & & \\
Not specified & $1(4 \%)$ & & & & \\
\hline
\end{tabular}


What are the measured outcomes on CSCL in initial VET (focus of analysis, forms of collaboration)?

Focus of analysis: Most studies focused on describing and analyzing learning processes $(13 ; 50 \%)$. Half as many studies described the outcome (e.g. learning gains) (6;23\%). Seven studies included both process and outcome (27\%) (see Table 3 ).

Collaboration - Definition and description: All reviewed papers included a form of collaborative learning. However, it is of note that most papers do not provide a definition of their core term 'collaboration'. Out of 26 papers on CSCL in VET, only five papers provided a definition. In the five studies defining collaboration, there seems to be no shared definition of collaboration. In the reviewed papers, we can distinguish between 'collaboration' and collaborative learning'. Bromme (2000) defined collaboration as "negotiating common ground". Other papers used the term collaborative learning' instead. Kuriyama and Sakai (2007) described collaborative learning as "collaborative and mutually interdependent learning, where the learners help each other and fosters individual learning responsibility within the group's activities to realize group objectives", while Johnson and Johnson (1994) defined collaborative learning as "a learning methodology based on students working together as a group to accomplish shared learning goals rather than an individual student achieving learning goals alone". Two papers referred to a definition by Dillenbourg (1999), who defined collaborative learning as "building shared knowledge through group activities, in which the participants are committed to or engaged in shared goals and problem solving". Studies that did not define collaboration, referred to related processes such as interaction, knowledge sharing, elaborative questioning, explaining, and reasoning.

\section{Content analysis of findings: three emerging topic areas}

In the following, we introduce three topic areas that emerged from the papers, which are specific to vocational learning: 1) collaborative writing-to-learn, 2) game-like solutions (gamification) and simulations, and 3) tangible objects in combination with augmented reality. These three topic areas found in current CSCL research are grounded in identifying how technologies are used in the selected studies and their intersections with collaboration and expected outcomes. These topic areas arose from the professional specificity of the learning objective (general vs specific), the kind of technology used (novel vs existing) and the kind of design of the collaboration in the learning task.

In the first topic area, studies focused on collaborative writing-to-learn and existing everyday technologies (like weblogs and wikis) were used to test the effectiveness of specific pedagogical scenarios on peer writing and/or peer commenting. Namely, collaborative writing-to-learn (Galbraith, 1999; Hayes \& Flower, 1980), i.e. the use of writing to foster reflection on one's own (professional) experiences (Boscolo \& Mason, 2001; Kember et al., 1996; Tynjälä, Mason, \& Lonka, 2001) was applied. Two of the studies addressed the field of commerce, two healthcare professions, and one included both domains. In this first area, existing technologies were exploited for their ability to have peers compare themselves to each other through texts (e.g. Gavota et al., 2010). 
Contents are specific to the profession and objectives aim at developing declarative and procedural professional knowledge in the domain.

In the second topic area, gamification technologies were designed to enhance both discipline-independent skills (e.g. Hämäläinen \& Oksanen, 2012) and discipline-specific skills (e.g. Hämäläinen, 2011). In practice, simulations and game-like solutions offered quasi-authentic ways to practice a variety of collaboration skills, such as interprofessional task-solving between different professions (e.g. Hämäläinen \& De Wever, 2013). In these studies, solving problems usually involved interactions with others and was motivated by shared concerns and goals (Dillenbourg, Järvelä, \& Fischer, 2009; Littleton \& Mercer, 2013). On the other hand, we have to consider that disciplineindependent skills are not separate from content but always connected with discipline matter (Silva, Goel, \& Mousavidin, 2009). With discipline-specific technologies, the idea behind the design of most of these environments was typically to offer a virtual environment allowing students to practice work situations that would otherwise be almost impossible (e.g., practicing the danger of electrical shocks), or too expensive to arrange (e.g. Hämäläinen, 2011). Given that there are many discipline-related learning activities within VET, different technological applications can present opportunities for designing collaborative learning that directly meet the future workplace needs. Currently, in this simulation and game-based research, collaboration scripts (Kobbe et al., 2007) have often been integrated into the game story to induce and develop discipline-independent and discipline-specific skills. Thus, learning games were designed so that collaboration was needed to solve the proposed problem or situation - meaning that each member of the group playing the serious game had to give her own contribution to be successful, as is increasingly the case in many VET programs.

In the third topic area, tangible objects and augmented reality were developed to meet discipline-specific professional needs and specific learning goals (e.g. in the field of logistics, see Jermann et al., 2009). Collaborative problem-solving tasks were designed to support the learning of content that is not transferable to other professions.

Taken together, these three emerging areas suggest that CSCL can be an interesting framework to apply to initial VET, especially for the development of professional skills. Depending on the specificity of the learning objective(s), this can be done both with existing and novel technologies. In the following discussion section, we present additional insights that arose once we analyzed the three main components of our analysis (technology, collaboration, and learning) independently.

\section{Discussion}

Work environments are undergoing radical changes. The extent to which changes in global working life require collaboration skills is a new element in today's technologyenhanced work-settings (Tynjälä, Häkkinen, \& Hämäläinen, 2014). As a direct result, collaborative skills, practice-based interactions (e.g. Noble \& Billett, 2017) and technologies are increasingly important at the workplace. Students and apprentices need to learn how to operate in such changing and complex environments; VET systems are 
challenged to prepare them to develop skills and abilities on how to use and take advantage of various kinds of technologies (Harteis, 2018) The current review investigated the state-of-the-art of CSCL research in initial VET. This led us to identify three research questions (RQs 1-3), starting from an analysis of the general demographics of the selected studies and then leading to investigations of the different components of our topic, namely technologies, collaboration and learning.

Although the field of VET seems to be an interesting area for investigating how collaborative learning and technology can advance students' and apprentices' knowledge, there are surprisingly few CSCL papers focusing on initial VET. One important conclusion of our research is thus that VET remains an under-researched field of study in CSCL society. Most research in CSCL has been conducted in K12 and higher education contexts. The strength of our review is therefore that it clearly highlights the need for additional studies in initial VET contexts. Our final corpus included only 26 papers, most of which (more than 60\%) originated from two main research groups in Finland and Switzerland. The dominance of these research groups is partly due to the commitment of funding agencies. In the case of Switzerland, an extensive research program was launched in 2009 to investigate different aspects of VET. The research focus of these two groups also affected the selection of professional domains involved, which would otherwise be scattered across a wider spectrum.

With regard to our review, the following limitations need to be considered. First, the lack of an extensive corpus of studies may be due to our restriction criteria related to the language of publication: having included only research published in English might explain the absence of studies from countries with a long tradition in VET but only publishing in their original language. It was surprising, in fact, that our systematic literature search did not lead to papers from Germany. Second, the restriction to initial VET has excluded research conducted on general vocational education, higher professional and tertiary vocational education, such as medical and teacher training. Third, we analyzed research focusing on collaborative technology, with the consequence of excluding other technologies, such as online learning journals for individual use. Future research could address these limitations by conducting an extended fulltext search in different languages, by including additional databases, and by extending the search terms to vocational education in general (instead of focusing on initial VET) and professional education. Different or extended selection criteria could lead to a wider corpus of papers. Our selection criteria and coding scheme allowed us to answer our specific research questions (demographics, methodologies, measured outcomes, and research topic areas). Future research could extend this work by asking additional, different research questions. One suggested area of research would be a comparison between the used technology, the vocational context, and the educational activity.

Despite these limitations, our study has several strengths. First, we focused on the technological component. The typical challenge in this respect was to find ways to make use of the added values of technologies as grounds for developing skills and abilities needed in vocational learners' workplace. Technology enabled new kinds of activities to supplement traditional vocational classroom and workplace practices. For example, mobile tools were introduced to bridge the physical boundaries of the school and workplaces 
(Cattaneo, Motta, \& Gurtner, 2015). 3D spaces provided safe environments to practice dangerous team-work practices e.g. at the construction sites (Hämäläinen, 2011). In our sample, technologies provided the possibility to deal with abstract complex tasks by reproducing concrete objects through tangibles and elaborating data provided by them through augmented reality (e.g. Schneider, Jermann, Zufferey, \& Dillenbourg, 2011). Moreover, technologies enabled the elaboration of group tasks to learn holistic work processes through simulating them in a secure, game-based environment (e.g. Hämäläinen \& Oksanen, 2012) or supported confrontation and the analysis of erroneous practice among peers using photos and writing (e.g. Gavota et al., 2010). In all cases, the specificity of technologies is also put to use in vocational education to integrate school- and workbased knowledge and to favor boundary crossing (Akkerman \& Bakker, 2011) across learning locations. Second, when focusing on collaboration, we noticed the absence of a common framework on how to design both collaboration and collaborative learning. Beyond CSCL, it seems that other frameworks are more central in VET research even when collaborative learning is used to design the studies. This leads to the hypothesis that a more specific framework for CSCL in VET is still needed and that studies specifically addressing the topic of collaboration and collaborative learning in initial VET are necessary. One could argue that no specific framework for CSCL in VET is required; however, collaboration is analyzed mostly at the learner level, and therefore interpreted as a process taking place among students without involving other VET-stakeholders, such as teachers and in-company supervisors. Specifically, this combination of schooland work-based actors (teachers and supervisors) and actions (intertwining learning activities at both locations) is what makes VET unique as a specific field. In our opinion, current research and practice often neglect the possibilities/opportunities to investigate the role of collaboration in vocational education, where the interaction between people in different locations is fundamental for the effective functioning of the (dual) systems.

Third, with respect to learning, we observed that only a minority of studies investigated both the processes and the products of learning. It is much more common to focus on only one or the other. Additionally, learning is often conceived as self-regulation and reflection. Although collaboration is always investigated from the learner's perspective, as highlighted before, the dynamics of learning are often examined from the teacher's perspective: it is not by chance that papers can be associated based on frameworks like teacher orchestration, scaffolding or scripting.Finally, we identified topic areas in research applying collaborative technologies for initial vocational education (RQ4). All three emerging topic areas showcased different uses of collaborative technologies for initial VET. Learners can learn collaboratively through writing activities, game-like learning environments, simulations, and augmented realities. To successfully apply each topic area, researchers needed to identify the specific characteristics of each initial VET context. On the technological side, these topic areas were applied with existing or novel technologies. The CSCL framework guided the implementation of these topic areas by providing a structure for the design of the technology, the form of collaboration, learning activities (input), learning objectives, and learning gains (outcome). This supports our perception that CSCL can serve as a useful framework for the design of studies in the field of initial, or also other forms of, VET. 


\section{Conclusions}

Taken together, our results suggest the existence of many opportunities for establishing a specific field of research on CSCL in initial VET. On the one hand, it can benefit from investigating the intersections between vocational practices, learning specific workrelated skills (motor skills, factual knowledge, procedural knowledge) and general skills (soft skills, social and communication skills). On the other hand, a theory-based, VETspecific framework needs to be developed that informs technology design and its crossings with collaboration and learning processes. Initial VET is a fascinating field of research, with many implications, both for educational and professional contexts. Nevertheless, the CSCL community has just begun to notice the potentials it can offer. We hope that the current review can inform further research by identifying possible streams of research and fostering CSCL VET research in the upcoming years.

\section{References}

Akkerman, S. F., \& Bakker, A. (2011). Boundary Crossing and Boundary Objects. Review of Educational Research, 81(2), 132-169.

Arvaja, M., Salovaara, H., Häkkinen, P., \& Järvelä, S. (2007). Combining individual and group-level perspectives for studying collaborative knowledge construction in context. Learning and Instruction, 17(4), 448-459.

Aveyard, H. (2010). Doing a literature review in health and social care: A practical guide. Maidenhead, UK: McGraw-Hill.

Beers, P. J., Kirschner, P. A., Boshuizen, H. P. A., \& Gijselaers, W. H. (2007). ICTsupport for grounding in the classroom. Instructional Science, 35(6), 535-556.

Billett, S. (2014). Integrating learning experiences across tertiary education and practice settings: A socio-personal account. Educational Research Review, 12, 1-13.

Boldrini, E., \& Cattaneo, A. (2014). Scaffolding Collaborative Reflective Writing in a VET Curriculum. Vocations and Learning, 7(2), 145-165.

Boscolo, P., \& Mason, L. (2001). Writing to Learn, Writing to Transfer. In P. Tynjälä, L. Mason, \& K. Lonka (Eds.), Writing as a Learning Tool (pp. 83-104). Springer Netherlands.

Braun, V., \& Clarke, V. (2006). Using thematic analysis in psychology. Qualitative Research in Psychology, 3(2), 77-101.

Bromme, R. (2000). Beyond one's own perspective: the psychology of cognitive interdisciplinarity. In P. Weingart \& N. Stehr (Eds.), Practicing interdisciplinarity (pp. 115-133). Toronto, Canada: University of Toronto Press.

Cattaneo, A. A. P., Motta, E., \& Gurtner, J.-L. (2015). Evaluating a Mobile and Online System for Apprentices' Learning Documentation in Vocational Education: Usability, Effectiveness and Satisfaction. International Journal of Mobile and Blended Learning (IJMBL), 7(3), 40-58.

Chanchalor, S., \& Kammeungmai, D. (2011). Vocational students in Thailand: collaborative learning via the Internet in groups of different sizes. Global Journal of En- 
gineering Education. King Mongkut's University of Technology Thonburi, Bangkok, Thailand, 13(2).

Cress, U., Stahl, G., Ludvigsen, S., \& Law, N. (2015). The Core features of CSCL: Social situation, collaborative knowledge processes and their design. International Journal of Computer-Supported Collaborative Learning, 10(2), 109-116.

Cuendet, S., Dehler-Zufferey, J., Ortoleva, G., \& Dillenbourg, P. (2015). An integrated way of using a tangible user interface in a classroom. International Journal of Computer-Supported Collaborative Learning, 10(2), 183-208.

Dillenbourg, P. (1999). What do you mean by 'collaborative learning'? In P. Dillenbourg (Ed.), Collaborative learning: Cognitive and computational approaches (pp. 1-16). Amsterdam: Pergamon, Elsevier Science.

Dillenbourg, P., Järvelä, S., \& Fischer, F. (2009). The Evolution of Research on Computer-Supported Collaborative Learning. In N. Balacheff, S. Ludvigsen, T. de Jong, A. Lazonder, \& S. Barnes (Eds.), Technology-Enhanced Learning (pp. 3-19). Springer Netherlands.

Galbraith, D. (1999). Writing as a knowledge-constituting process. Knowing What to Write: Conceptual Processes in Text Production, 4, 139-164.

European Commission (2013): Work-based learning in Europe - Practices and Policy Pointers. Retrieved from http://ec.europa.eu/education/lifelong-learning-policy/do c/work-based-lea rning-in-europe_en.pdf.

Eteläpelto, A. (2008). Perspectives, prospects and progress in work-related learning. In S. Billett, C. Harteis, \& A. Eteläpelto (Eds.), Emerging perspectives of workplace learning (pp. 233-247). Rotterdam: Sense Publishers.

Gavota, M. C., Cattaneo, A., Arn, C., Boldrini, E., Motta, E., Schneider, D., \& Betrancourt, M. (2010). Computer-supported peer commenting: a promising instructional method to promote skill development in vocational education. Journal of Vocational Education \& Training, 62(4), 495-511.

Harteis, C. (2018). Machines, Change and Work: An Educational View on the Digitalization of Work. In The Impact of Digitalization in the Workplace (pp. 1-10). Springer, Cham.

Hämäläinen, R. (2008). Designing and investigating pedagogical scripts to facilitate computer-supported collaborative learning. Retrieved from: https://jyx.jyu.fi/ds pace/handle/123456789/37597

Hämäläinen, R. (2011). Using a game environment to foster collaborative learning: a designbased study. Technology, Pedagogy and Education, 20(1), 61-78.

Hämäläinen, R., \& Cattaneo, A. (2015). New TEL Environments for Vocational Education - Teacher's Instructional Perspective. Vocations and Learning, 8(2), 135-157.

Hämäläinen, R., Cincinnato, S., Malin, A., \& De Wever, B. (2014). VET workers' problem-solving skills in technology-rich environments: European approach. International Journal For Research In Vocational Education And Training, 1(1), 57-80. doi:10.13152/IJRVET.1.1.4

Hämäläinen, R., De Wever, B., Malin, A. \& Cincinnato, S. (2015). Education and working life: VET adults' problem-solving skills in technology-rich environments. Computers \& Education, 88, 38-47. 
Hämäläinen, R., \& De Wever, B. (2013). Vocational education approach: New TEL settings - new prospects for teachers' instructional activities? International Journal of Computer-Supported Collaborative Learning, 8(3), 271-291.

Hämäläinen, R., Lanz, M., \& Koskinen, K. T. (2018). Collaborative Systems and Environments for Future Working Life: Towards the Integration of Workers, Systems and Manufacturing Environments. In The Impact of Digitalization in the Workplace (pp. 25-38). Springer, Cham.

Hämäläinen, R., \& Oksanen, K. (2012). Challenge of supporting vocational learning: Empowering collaboration in a scripted 3D game-How does teachers' realtime orchestration make a difference? Computers $\&$ Education. Retrieved from http://www.sciencedirect.com/science/article/pii/S0360131512000036

Hämäläinen, R., \& Oksanen, K. (2013). Collaborative 3D learning games for future learning: teachers' instructional practices to enhance shared knowledge construction among students. Technology Pedagogy and Education, 23(1), 81-101.

Hämäläinen, R., Oksanen, K., \& Häkkinen, P. (2008). Designing and analyzing collaboration in a scripted game for vocational education. Computers in Human Behavior, 24(6), 2496-2506.

Hayes, J. R., \& Flower, L. (1980). Identifying the organization of writing processes.

Inayat, I., ul Amin, R., Inayat, Z., \& Salim, S. S. (2013). Effects of Collaborative Web Based Vocational Education and Training (VET) on Learning Outcomes. Computers E Education, 68, 153-166.

Jermann, P., Zufferey, G., Schneider, B., Lucci, A., Lépine, S., \& Dillenbourg, P. (2009). Physical Space and Division of Labor Around a Tabletop Tangible Simulation. In Proceedings of the 9th International Conference on Computer Supported Collaborative Learning - Volume 1 (pp. 345-349). Rhodes, Greece: International Society of the Learning Sciences.

Johnson, D.W., and R.T. Johnson. 1994. Learning together and alone: Cooperative, competi- tive and individualistic learning. Englewood Cliffs, NJ: Prentice-Hall.

Kember, D., Jones, A., Loke, A., McKay, J., Sinclair, K., Tse, H., Yeung, E. (1996). Encouraging Critical Reflection Through Small Group Discussion of Journal Writing. Innovations in Education 83 Training International, 33(4), 213-220.

Kitchenham, B. \& Charters, S. (2007), Guidelines for performing systematic literature reviews in software engineering, Technical report, Keele University (UK).

Kobbe, L., Weinberger, A., Dillenbourg, P., Harrer, A., Hämäläinen, R., Häkkinen, P., \& Fischer, F. (2007). Specifying computer-supported collaboration scripts. International Journal of Computer-Supported Collaborative Learning, 2(2-3), 211-224.

Kuriyama, K., \& Sakai, K. (2007, September). An Agent Oriented Environment for Collaborative Learning-Lessons Learned Through Vocational Training on Software Design with UML. In International Conference on Knowledge-Based and Intelligent Information and Engineering Systems (pp. 567-574). Springer, Berlin, Heidelberg.

Lee, D., Huh, Y., \& Reigeluth, C. M. (2015). Collaboration, intragroup conflict, and social skills in project-based learning. Instructional Science, 43(5), 561-590.

Littleton, K., \& Mercer, N. (2013). Interthinking: Putting Talk to Work. Taylor \& Francis. 
Noble, C., \& Billett, S. (2017). Learning to prescribe through coworking: junior doctors, pharmacists and consultants. Medical Education, 51(4), 442-451.

Maclean, R., \& Wilson, D. (Eds.). (2009). International handbook of education for the changing world of work: Bridging academic and vocational learning (Vol. 1). Springer Science \& Business Media.

Minnaert, A., Boekaerts, M., de Brabander, C., \& Opdenakker, M.-C. (2011). Students' Experiences of Autonomy Competence, Social Relatedness and Interest Within a CSCL Environment in Vocational Education: The Case of Commerce and Business Administration. Vocations and Learning, 4(3), 175-190.

Motta, E., Boldrini, E., \& Cattaneo, A. (2013). Technologies to "Bridge the Gap" among Learning Contexts in Vocational Training. In Didactic Strategies and Technologies for Education (pp. 247-265). IGI Global.

Oksanen, K., \& Hämäläinen, R. (2012). Collaborative serious game as a sociable computer-supported collaborative learning environment. In Proceedings of the 6th European conference on game based learning (pp. 364-371). Academic Publishing International Reading, UK.

Oksanen, K., \& Hämäläinen, R. (2013a). Perceived Sociability and Social Presence in a Collaborative Serious Game. International Journal of Game-Based Learning, 3(1), $34-50$.

Oksanen, K., \& Hämäläinen, R. (2013b). Supporting and Facilitating Collaborative Learning in Serious Games. In European Conference on Games Based Learning (p. 683). Academic Conferences International Limited.

Oksanen, K., \& Hämäläinen, R. (2014). Game Mechanics in the Design of a Collaborative 3D Serious Game. Simulation \& Gaming, 45(2), 255-278.

Ortoleva, G., \& Bétrancourt, M. (2015). Collaborative writing and discussion in vocational education: Effects on learning and self-efficacy beliefs. Journal of Writing Research, 7(1), 95-122.

Partnership for 21st Century Skills. (2016, November 21). Retrieved from http://www.p 21.org/our-work/p21-framework

Qin, Z., Johnson, D. W., \& Johnson, R. T. (1995). Cooperative versus competitive efforts and problem solving. Review of Educational Research, 65(2), 129-143.

Sanz, M. (2010). New teaching experiments for new learning strategies how design-based research can increase efficiency in education. In Proceedings of the WMSCI 2010 The 14th World Multi-Conference on Systemics, Cybernetics and Informatics (p. 6). WMSCI.

Schneider, B., Jermann, P., Zufferey, G., \& Dillenbourg, P. (2011). Benefits of a Tangible Interface for Collaborative Learning and Interaction. IEEE Transactions on Learning Technologies, 4(3), 222-232.

Silva, L., Goel, L., \& Mousavidin, E. (2009). Exploring the dynamics of blog communities: the case of MetaFilter. Information Systems Journal, 19(1), 55-81.

Stahl, G., Koschmann, T., \& Suthers, D. (2006). Computer-supported collaborative learning: A historical perspective. In R. K. Sawyer (Ed.), Cambridge handbook of the learning sciences (pp. 406-427). Cambridge: Cambridge University Press.

Stamm, M. (2007). Kluge Köpfe, goldene Hände: überdurchschnittlich begabte Lehrlinge 
in der Berufsbildung: Rüegger.

Strahm, R. H. (2016). Why University Education is not the only path forward and the apprenticeship is rated at the top. In R. H. Strahm, B. H. Geiger, C. Oertle, \& E. Swars (Eds.), Vocational and Professional Education and Training in Switzerland. Success factors and challenges for sustainable implementation abroad (pp. 19-50). Bern: hep Verlag.

Taylor, A., \& Freeman, S. (2011). 'Made in the trade': youth attitudes toward apprenticeship certification. Journal of Vocational Education 83 Training, 63(3), 345-362.

Tielman, K., den Brok, P., Bolhuis, S., \& Vallejo, B. (2012). Collaborative learning in multicultural classrooms: a case study of Dutch senior secondary vocational education. Journal of Vocational Education \& Training, 64(1), 103-118.

Trilling, B., \& Fadel, C. (2009). 21st Century Skills: Learning for Life in Our Times. Wiley.

Tynjälä, P., Häkkinen, P. \& Hämäläinen R., (2014). TEL@work - towards integration of theory and practice. British Journal of Educational Technology. 45(6), 990-1000.

Tynjälä, P., Mason, L., \& Lonka, K. (2001). Writing as a Learning Tool: An Introduction. In P. Tynjälä, L. Mason, \& K. Lonka (Eds.), Writing as a Learning Tool (pp. 7-22). Springer Netherlands.

Valtonen, T., Hacklin, S., Dillon, P., Vesisenaho, M., Kukkonen, J., \& Hietanen, A. (2012). Perspectives on personal learning environments held by vocational students. Computers \& Education, 58(2), 732-739.

White, B. (2010). Using ICT to enhance curriculum opportunities for students in rural and remote schools. Australian Educational Computing, 25(2), 27.

Yang, Y.-T. C. (2015). Virtual CEOs: A blended approach to digital gaming for enhancing higher order thinking and academic achievement among vocational high school students. Computers \& Education, 81, 281-295.

Zihlmann, R., \& Jungo, D. (2015). Berufswahl in Theorie und Praxis. SDBB.

\section{Biographical Notes}

Dr Beat A. Schwendimann is a learning scientist interested in how different forms of technologies and knowledge representations facilitate knowledge integration and collaborative forms of learning. He conducted his Ph.D. research as a Fulbright scholar at the University of California, Berkeley. His postdoctoral work at the University of Sydney and at EPFL investigated how innovative technologies can support collaborate processes of groups of educational designers and, respectively, vocational students. He currently serves on the executive board of the Swiss teacher federation $(\mathrm{LCH})$, where he heads the pedagogy expert group.

Dr Bram De Wever is associate professor at the Department of Educational Studies at Ghent University, Belgium and head of the research group TECOLAB. His research is focusing on technology enhanced learning and instruction, peer assessment and feedback, computer-supported collaborative learning activities, professional development, inquiry 
learning in higher and adult education settings, and learning design for hands-on adult learners. He is also teaching courses on adult education, higher education, and powerful learning environments.

Full Professor Raija Hämäläinen works in the field of technology-enhanced learning at the Center for Research for Learning and Teaching at the University of Jyväskylä, Finland. Her research interests include collaborative learning, workplace-learning and vocational education and training (VET). She has designed a long-term research line that focuses on designing and investigating new learning environments for future educational efforts. She is an associate editor of Educational Research Review.

Dr Alberto A. P. Cattaneo is professor and head of the research field "Innovations in Vocational Education" at the Swiss Federal Institute for Vocational Education and Training (SFIVET), Switzerland. His actual main research fields concern the integration of ICT in teaching-and-learning processes, reflective learning in VET, multimedia learning especially when it comes to using interactive videos, teacher education and their professional competence development. 